\title{
Principal components analysis of a large cohort with Tourette syndrome
}

\author{
Mary M. Robertson, Robert R. Althoff, Adam Hafez and David L. Pauls
}

\section{Background}

Tourette syndrome is a heterogeneous familial disorder for which the genetic mechanisms are unknown. A better characterisation of the phenotype may help identify susceptibility genes.

\section{Aims \\ To extend previous factor-analytic studies of the syndrome.}

\section{Method}

Symptom data from 410 people with Tourette syndrome were included in agglomerative hierarchical cluster and principal components analyses.

\section{Results}

Five factors were observed, characterised by: (1) socially inappropriate behaviours and other complex vocal tics; (2) complex motor tics; (3) simple tics; (4) compulsive behaviours; and (5) touching self. Individuals with cooccurring attention-deficit hyperactivity disorder had significantly higher factor scores on Factors 1 and 3 , whereas individuals with co-occurring obsessive-compulsive disorder and behaviours had significantly higher factor scores for Factors $1-4$

\section{Conclusions}

These findings add to the growing body of evidence that Tourette syndrome is not a unitary condition and can be disaggregated into more homogeneous symptom components.

\section{Declaration of interest}

None. Funding detailed in Acknowledgements.
Tourette syndrome (Tourette disorder, DSM-IV-TR) is characterised by the presence of multiple motor and one or more vocal/phonic tics present for more than a year. ${ }^{1,2}$ Tics wax and wane over time and do not necessarily occur concurrently. The diagnosis of Tourette syndrome is relatively straightforward, but there is considerable clinical variability across individuals. The importance of evaluating the individual elements of a complex disorder such as Tourette syndrome cannot be overstated in terms of research into the underpinnings of the disorder and its clinical course. Although the familial nature of Tourette syndrome and chronic tics has been well-documented, ${ }^{3}$ and twin studies implicate genetic and non-genetic factors in the expression of Tourette syndrome, ${ }^{4,5}$ specific, replicable findings for genetic loci have been somewhat elusive. To date, ten genetic linkage/association studies have been published. ${ }^{6-15}$ Regions of interest have been reported for chromosomes 2, 3, 4, 5, 8, 9, 10,11, 13, 17 and 19. All of these findings are potentially very important, but none has yet been replicated. Given strong evidence that Tourette syndrome is genetic and the lack of significant replicable linkage, combined with association findings showing that the underlying genetic mechanisms are complex and likely to be heterogeneous, the question arises as to whether there may be a way to disaggregate this heterogeneity to allow for clearer targets for clinical and genetic research.

Investigators have employed a variety of methods that address multiple quantitative phenotypic dimensions, making it possible to examine distinct components of complex phenotypes. Studies of reading disability, ${ }^{16}$ attention-deficit hyperactivity disorder (ADHD), ${ }^{17,18}$ obsessive-compulsive disorder (OCD $)^{19}$ and autism ${ }^{20}$ have used various methods to discern possible underlying phenotypic constructs. There have been few attempts to formally classify people with Tourette syndrome on the basis of their tic phenomenology. ${ }^{21,22}$ Robertson et al $^{22}$ reported that coprolalia and echophenomena were related to obsessional symptoms and increased severity. To date, three cluster and/or factor analytic investigations of tic phenomenology have been undertaken. ${ }^{23-25}$
Alsobrook \& Pauls ${ }^{23}$ conducted a principal components factor analysis on a cohort of 85 individuals with Tourette syndrome and reported four factors: Factor 1 (APF1) was characterised by behaviours that included coprolalia, 'aggressive' and self-injurious behaviours; Factor 2 (APF2) was characterised by simple and complex motor tics and simple vocal/phonic tics (e.g. noises, but no actual words); Factor 3 (APF3) was characterised by 'compulsive-like' behaviours such as forced touching, repetitive behaviours, echo- and paliphenomena; and Factor 4 (APF4) was characterised by the absence of grunting tics and the presence of finger and hand tapping that was distinguished from finger and hand tics or forced touching. Robertson \& Cavanna ${ }^{25}$ reported the results of a principal components factor analysis completed on 69 individuals with Tourette syndrome and chronic tics, all of whom were members of a large extended Tourette syndrome pedigree. ${ }^{26}$ It should be noted that these investigators included symptoms of other psychopathology (that are generally not considered to be tics, such as symptoms of inattention, hyperactivity, obsessionality, compulsivity, depression and anxiety) in their principal components factor analysis. Three factors were observed: Factor 1 (RCF1) consisted of predominantly 'pure tics' (both simple and complex motor and vocal/phonic tics); Factor 2 (RCF2) included 'ADHD and aggressive behaviours', and complex motor and vocal/phonic tics, including coprophenomena; and Factor 3 (RCF3) was characterised by 'depression-anxiety-obsessional symptoms and self-injurious behaviour' and compulsive-like tics, including counting and 'evening-up'. Finally, Mathews et $a l^{24}$ reported results of hierarchical agglomerative cluster analysis (HACA) from two genetically isolated populations (Costa Ricans and Ashkenazi Jews) consisting of 254 individuals with Tourette syndrome. These investigators observed two clusters which were essentially the same in the two genetic isolates: one characterised by simple motor and vocal/phonic tics and the other by complex motor and vocal/phonic tics. Thus, the results of all of these studies suggest that Tourette syndrome is not a unitary disorder and can be disaggregated into more homogeneous components. 
However, these studies had relatively small samples for principal components analyses. Furthermore, one study included only related individual $\mathrm{s}^{25}$ and another ${ }^{24}$ examined genetically isolated populations. All of these call into question the generalisablity of their results and speak to the need for a larger study of singleton individuals with the syndrome.

The primary aim of the current investigation was to identify quantitative components of Tourette syndrome symptomatology using a large, well-characterised sample of singleton individuals with the syndrome. As noted by Verkerk et $a l^{27}$ it is crucial in future genetic studies of Tourette syndrome to search for more homogeneous phenotypes of the syndrome. These investigators suggest that the use of factor-analysed quantitative symptom scores might prove useful. If such components can be identified and can be examined in genetically informative data-sets, it could lead to a major advance in future studies of Tourette syndrome.

\section{Methods}

\section{Participants}

The sample for this study consisted of 410 people with Tourette syndrome $(75.9 \%$ male) who were seen at the National Hospital for Neurology and Neurosurgery (NHNN), Queen Square, London, UK. This research was approved by the ethics committee at the NHNN for assessing both children and adults. Participants ranged in age from 3 to 59 years with a mean of 20.4 (s.d.=12.3), All individuals over the age of 18 years gave informed consent. For those individuals under the age of 18 , parents gave informed consent for the children to be part of the study. All individuals were assessed and/or diagnosed with Tourette syndrome by the first author (M.M.R.) using DSM-III ${ }^{28}$ or DSM-IV-TR ${ }^{2}$ criteria

\section{Measures}

The National Hospital Interview Schedule (NHIS) ${ }^{29}$ was used to characterise the motor and vocal/phonic tics. The NHIS is a semi-structured diagnostic interview shown to be reliable and valid that allows for the collection of detailed information on over 100 specific motor and vocal/phonic tics in 32 different categories. For example, specific content is collected for coprolalic utterances as well as other vocal/phonic tics. In addition, Yale Global Tic Severity Scale (YGTSS) ${ }^{30}$ scores were obtained from 402 individuals. Percentage scores on the YGTSS ranged from 3 to $100 \%$ with a mean of $44.6 \%$ (s.d. $=22.2$ ), covering the entire range of severity, but with the mean in the moderate range. Additional information was obtained through diagnostic interview concerning age at onset of motor and vocal/phonic tic symptoms and co-occurring DSM-IV diagnoses including ADHD, substance misuse and OCD. Moreover, data were coded for the presence or absence of obsessive-compulsive behaviours without full-criterion OCD, self-injurious behaviour and aggression, along with the presence or absence of a family history of Tourette syndrome or chronic tics, ADHD and/or OCD.

\section{Statistical analysis}

All analyses were performed using SPSS 15.0 for Windows. Cluster analyses were initially performed to determine whether any of the dichotomous symptoms could be combined into pseudocontinuous clusters. Here, as in previous studies of people with Tourette syndrome, ${ }^{23-25}$ tic symptoms were combined into pseudo-continuous measures by performing an initial data reduction of the entire array of dichotomous symptom variables into clusters. This reduction was achieved by an $\mathrm{HACA}^{31}$ analysis on 32 tic symptom categories obtained from the NHIS. The
HACA method progressively combines variables into related clusters until all variables are subsumed into a single cluster, using the average linkage between-groups method to evaluate the Euclidean-squared cluster distances. The HACA does not rely on preconceived characterisations concerning the number of clusters (such as specifying the number of clusters) or the relationships between them (such as specifying the distances between clusters). The stages of agglomeration are displayed as a dendrogram, with the formation of clusters at each stage plotted along a scaled stage distance axis. For the current study, the best set of clusters required to adequately represent the data was determined by inspection of the dendrogram. There is no test of significance for clustering results other than their use in subsequent analyses. For each resulting cluster, a score was then generated for each participant equal to the sum of the symptom variables contained therein: symptom variables were scored 0 for never present and 1 for ever present.

The resultant cluster scores were then used as input variables for the principal components factor analysis. Principal components factor analyses are typically based upon non-dichotomous variables; however, the variables that did not fall into a cluster on the dendrogram were entered as dichotomous variables. The use of dichotomous variables can be justified in exploratory approaches such as reported here. Using Kaiser-Guttman's rule, after factor extraction promax rotation was performed on factors with eigenvalues greater than 1.0 (factors with eigenvalues less than 1.0 are generally spurious and non-reproducible) and compared. This procedure minimised the number of variables with high loadings on each extracted factor and allowed for a more straightforward interpretation; other rotations were not explored. Symptom loadings, with coefficient absolute values greater than 0.375 , were used to describe the factors.

Relations among extracted factors, age at onset of tics, gender, and co-occurring symptoms or disorders were then examined by comparing the mean factor score on each factor with each variable. Pearson correlations were computed for age at onset of the vocal/phonic and motor tics compared with factor scores for each factor. Because this resulted in 15 significance tests, a $P$-value of $<0.003$ was considered to be significant. Relations between factors and co-occurring disorders were made by comparing mean factor scores of individuals with and without the co-occurring diagnoses. Because this examination resulted in 25 significance tests, a $P$-value of $<0.002$ was considered to be significant.

\section{Results}

Heirarchical agglomerative cluster analysis on the 32 tic symptoms identified 7 clusters of symptoms; 13 symptoms remained as lone variables but were treated as clusters in subsequent analyses. The dendrogram illustrating the progression of cluster formation and the final resulting clusters is shown in online Fig. DS1. The clusters were characterised by the following behaviours: tics of the head and face, including throat clearing and shoulder shrugging; touching self; leg and foot movements; grunting; hopping, skipping, and jumping; forced touching; coughing; arm movements; adjusting clothing and compulsive looking; coprolalia and copropraxia, hitting, palipraxia, kicking and mental coprolalia; spitting; palilalia; echolalia and echopraxia; self-injurious behaviour; random words; tensing body; tensing abdomen; touching chin to chest and shoulders; torso and hip movements; and finger tapping.

For each of these clusters, a score was generated equal to the sum of the elemental symptom variables. These scores were then included in the factor analysis. After factor extraction and promax 
rotation of the cluster variables, five factors resulted which accounted for $46.6 \%$ of the symptomatic variance in the sample (see online Table DS1). Factor 1, which accounted for $22.9 \%$ of the variance, includes coprolalia, copropraxia, echolalia, echopraxia, palilalia, palipraxia, hitting, spitting, kicking, random words, forced touching and self-injurious behaviour. Factor 2, which accounted for $7.4 \%$ of the variance, is characterised by complex motor tics (e.g. arm, leg, foot movements, hopping, skipping, jumping and torso movements). Factor 3, which accounted for $5.8 \%$ of the variance, includes coughing, tensing of the body, grunting and simple motor and vocal/phonic tics (e.g. eye blinking, facial tics, head tics, noises, and throat clearing). Factor 4, which accounted for $5.4 \%$ of the variance is characterised by compulsive-like behaviours such as repetitive looking, adjusting clothing, finger tapping, leg and foot movement, and tensing of the abdomen. Finally, Factor 5, which accounted for $5.1 \%$ of the variance, also included simple motor and vocal/phonic tics as well as touching one's self.

In an attempt to provide evidence for the external validity of these factors, additional analyses were undertaken in which the relationship between these five factors and age at onset, gender, the presence of co-occurring conditions (e.g. ADHD, OCD, substance misuse, self-injurious behaviour and aggression) and the presence of a positive family history for Tourette syndrome, OCD/obsessive-compulsive behaviour and ADHD was examined. The results are presented in Tables 1-5. All comparisons were corrected for multiple testing. There were significant negative correlations between Factor 1 and Factor 3 scores and age at onset

\begin{tabular}{|c|c|c|c|c|c|}
\hline & Factor 1 & Factor 2 & Factor 3 & Factor 4 & Factor 5 \\
\hline \multicolumn{6}{|c|}{ Age at onset of phonic tics } \\
\hline$r$ & -0.173 & -0.011 & -0.123 & -0.070 & -0.102 \\
\hline$P$ & $<0.001$ & NS & NS & NS & NS \\
\hline \multicolumn{6}{|c|}{ Age at onset of motor tics } \\
\hline$r$ & -0.177 & -0.051 & -0.179 & -0.078 & -0.060 \\
\hline$P$ & $<0.001$ & NS & $<0.001$ & NS & NS \\
\hline \multicolumn{6}{|c|}{ Age at onset } \\
\hline$r$ & -0.177 & -0.086 & -0.168 & -0.097 & -0.097 \\
\hline P & $<0.0001$ & NS & $<0.001$ & NS & NS \\
\hline
\end{tabular}

\begin{tabular}{|c|c|c|c|}
\hline Factor & Factor score $\leqslant 0$ & Factor score $>0$ & Significance \\
\hline 1 & $7.40(n=212)$ & $6.29(n=189)$ & $P<0.005$ \\
\hline 2 & $7.25(n=196)$ & $6.52(n=205)$ & NS \\
\hline 3 & $7.39(n=185)$ & $6.43(n=216)$ & $P<0.015$ \\
\hline 4 & $7.17(n=205)$ & $6.57(n=196)$ & NS \\
\hline 5 & $7.18(n=187)$ & $6.61(n=214)$ & NS \\
\hline
\end{tabular}

\begin{tabular}{|c|c|c|c|c|c|}
\hline Co-occurring diagnosis & Factor 1 & Factor 2 & Factor 3 & Factor 4 & Factor 5 \\
\hline \multicolumn{6}{|l|}{ ADHD } \\
\hline No $(n=157)$ & -0.331 & 0.058 & -0.230 & -0.149 & -0.082 \\
\hline Yes $(n=230)$ & 0.255 & 0.060 & 0.167 & 0.118 & 0.054 \\
\hline$P$ & $<0.0001$ & NS & $<0.0001$ & $N S^{a}$ & \\
\hline \multicolumn{6}{|c|}{ OCD/obsessive-complusive behaviour } \\
\hline No $(n=68)$ & -0.749 & -0.298 & -0.594 & -0.494 & -0.172 \\
\hline Yes $(n=341)$ & 0.147 & 0.059 & 0.120 & 0.100 & 0.034 \\
\hline$P$ & $<0.0001$ & $<0.007$ & $<0.0001$ & $<0.0001$ & NS \\
\hline \multicolumn{6}{|l|}{ Substance misuse } \\
\hline No $(n=316)$ & -0.025 & -0.009 & -0.009 & -0.046 & -0.043 \\
\hline Yes $(n=85)$ & 0.087 & 0.082 & 0.008 & 0.191 & 0.121 \\
\hline$P$ & NS & NS & NS & NS & NS \\
\hline \multicolumn{6}{|l|}{ Self-injurious behaviour } \\
\hline No $(n=232)$ & -0.368 & 0.043 & -0.087 & -0.448 & 0.008 \\
\hline Yes $(n=177)$ & 0.479 & -0.056 & 0.117 & 0.590 & -0.113 \\
\hline$P$ & $<0.0001$ & NS & NS & $<0.0001$ & NS \\
\hline \multicolumn{6}{|l|}{ Aggression } \\
\hline No $(n=223)$ & -0.246 & 0.049 & -0.016 & -0.115 & -0.128 \\
\hline Yes $(n=181)$ & 0.297 & -0.094 & 0.007 & 0.130 & 0.141 \\
\hline$P$ & $<0.0001$ & NS & NS & NS & -0.007 \\
\hline
\end{tabular}




\begin{tabular}{|c|c|c|c|c|c|}
\hline Family history & Factor 1 & Factor 2 & Factor 3 & Factor 4 & Factor 5 \\
\hline \multicolumn{6}{|c|}{ Tourette syndrome } \\
\hline No $(n=85)$ & -0.063 & -0.050 & 0.042 & -0.168 & 0.053 \\
\hline Yes $(n=310)$ & 0.013 & 0.019 & 0.009 & 0.071 & -0.011 \\
\hline$P$ & NS & NS & NS & NS & NS \\
\hline \multicolumn{6}{|c|}{ OCD/obsessive-complusive behaviour } \\
\hline No $(n=120)$ & -0.090 & -0.009 & 0.013 & -0.117 & 0.015 \\
\hline Yes $(n=275)$ & 0.043 & 0.005 & 0.015 & 0.062 & -0.015 \\
\hline$P$ & NS & NS & NS & NS & NS \\
\hline \multicolumn{6}{|l|}{ ADHD } \\
\hline No $(n=266)$ & -0.106 & -0.017 & -0.049 & -0.121 & -0.059 \\
\hline Yes $(n=124)$ & 0.189 & 0.054 & 0.129 & 0.268 & 0.179 \\
\hline$P$ & $<0.007$ & NS & NS & $<0.0001$ & NS \\
\hline
\end{tabular}

\begin{tabular}{|lccccc|}
\hline Table 5 & Mean factor scores for males and females & & & & \\
& Factor 1 & Factor 2 & Factor 3 & Factor 4 & Factor 5 \\
\hline Male $(n=312)$ & 0.031 & 0.086 & 0.074 & 0.063 & 0.143 \\
\hline Female $(n=98)$ & -0.101 & -0.276 & -0.235 & -0.200 & -0.454 \\
\hline$P$ & NS & $<0.002$ & $<0.007$ & NS & $<0.0001$ \\
NS, not significant. & & & & \\
\hline
\end{tabular}

(Factor 1: $r=-0.177, P<0.0001$; Factor 3: $r=-0.168, P<0.001$ ) (Table 1). When individuals were dichotomised according to whether their factor score was $\leqslant 0$ or $>0$, significant differences for age at onset were observed for Factors 1 and 3 (Table 2). The mean age at onset of Tourette syndrome for individuals with a score $>0$ for Factor 1 was 6.29 compared with the mean of 7.4 for individuals with a score of $\leqslant 0(P<0.005)$. For Factor 3, the mean age at onset for individuals with a factor score $>0$ was 6.43 compared with 7.39 for individuals with a score $\leqslant 0$ $(P<0.015)$.

The next set of analyses compared the mean factors scores for individuals with co-occurring ADHD, OCD or obsessivecompulsive behaviour, substance misuse, self-injurious behaviour and aggression (Table 3). For individuals with co-occurring ADHD, there were significant differences in mean factor scores observed for Factor $1 \quad(P<0.0001)$ and Factor $3(P<0.0001)$, and there was a trend for Factor $4(P<0.010)$ (note that a $P<0.003$ was required for statistical significance after correction for multiple comparisons). For individuals with OCD/obsessivecompulsive behaviour, there were significant differences in mean factor scores for Factors $1(P<0.0001), 2(P<0.007), 3(P<$ $0.0001)$ and $4(P<0.0001)$. For individuals with co-occurring substance misuse, no significant differences were observed. Individuals with self-injurious behaviour had significantly different mean factor scores for Factors $1(P<0.0001)$ and $4(P<0.0001)$. This was not surprising since both factors included self-injurious behaviour as a tic. Finally, individuals with aggression had mean factor score differences for Factors $1 \quad(P<0.0001)$ and 5 $(P<0.007)$.

With respect to family history of Tourette syndrome, OCD/ obsessive-compulsive behaviour and ADHD, there were few significant differences in mean factor scores (Table 4). The only significant differences observed were for individuals with ADHD. The mean factor scores for Factor 1 were significantly higher $(P<0.007)$ for individuals with both Tourette syndrome and a family history of ADHD, as were mean factor scores for Factor $4(P<0.0001)$. It should be noted that the majority of individuals in this study had positive family histories for both Tourette syndrome (79\%) and OCD/obsessive-compulsive behaviour
(71\%), thus the fact that neither showed any relation to any factors scores could reflect the fact that there was little variability in family history for both Tourette syndrome and OCD/obsessivecompulsive behaviour in this sample.

Finally, when males and females were compared (Table 5), females had lower scores for all five factors and the differences for Factors $2(P<0.002), 3(P<0.007)$ and $5(P<0.0001)$ were statistically significant.

\section{Discussion}

\section{A unitary disorder?}

The current findings are consistent with the growing body of evidence that Tourette syndrome is not a unitary condition as suggested by all international diagnostic criteria. Two investigations using principal components factor analysis ${ }^{23,25}$ and one study using $\mathrm{HACA}^{24}$ have shown that the categorical classification of Tourette syndrome using either ICD- $10^{1}$ or DSM-IV-TR ${ }^{2}$ criteria is likely to consist of several different components comprised of different types of tics and associated behaviours. This finding is supported by the current results. There are notable differences between the previous three studies and the current findings, and also significant similarities.

First, in the two previous principal components factor analysis studies pure tic factors (APF2 and RCF1) were observed that were distinct from the more complex compulsive-like tics that often occur in Tourette syndrome. The pure tic factors included both simple and complex tics. In contrast, in the current report when a promax rotation was implemented, two pure tic factors were observed; one included predominantly simple tics (Factor 3), both motor and vocal/phonic, whereas the other included predominantly complex motor tics (Factor 2). These results suggest that complex motor tics are separate from both simple motor and vocal/phonic tics as well as from complex vocal/phonic tics, a finding that is quite similar to the results reported by Mathews et $a l^{24}$ in that study, two clusters were identified, one characterised by simple tics, the other by complex tics. 
It is also noteworthy that in all three principal components factor analysis studies (the two previous ${ }^{23,25}$ and the current report) there were two additional factors observed: one characterised by what could be broadly termed 'aggressive behaviours', including coprophenomena, spitting, hitting and kicking, and the other characterised by more compulsive-like behaviours (APF1 and RCF2). The current results demonstrate a similar aggressive behaviour factor (Factor 1) and a factor with compulsive-like behaviour (Factor 4). Therefore, even with differences in samples and methodology, there are areas of convergence across these studies. All studies suggest that tics occurring in individuals with Tourette syndrome can be separated into two broad categories: one comprised of simple tics and the other complex tics. Furthermore, the principal components factor analysis studies suggest that these can be further broken down into a 'pure simple tic' factor, a 'pure complex motor tic' factor, a factor characterised by coprophenomena and aggressive behaviours, and another factor characterised by predominantly compulsive behaviours.

\section{Differences from previous studies}

There are a number of reasons why the results of the current study do not agree completely with previous studies. These include the fact that: (a) our study $(n=410)$ is considerably larger than any of the previous three studies; (b) the instruments used were different across the various studies. In the current study, the variables collected by the NHIS were grouped to be consistent with the instrument used in the Alsobrook \& Pauls study, ${ }^{23}$ although a few additional variables were included. Robertson \& Cavanna ${ }^{25}$ also used the NHIS, but augmented the data obtained with another structured interview (Schedule for Affective Disorders and Schizophrenia - Lifetime version) and many self-report scales for obsessionality, mood, and other psychopathology; and (c) the samples were drawn from different populations: one from a clinic in the $\mathrm{USA}^{23}$ another from a single large family ascertained through individuals with Tourette syndrome in the UK, ${ }^{25,26}$ another from two genetic isolates ${ }^{24}$ and the current one from a dedicated Tourette syndrome tertiary clinic in the UK. Furthermore, Robertson \& Cavanna ${ }^{25}$ included data about psychopathology in the principal components factor analysis, whereas the three other studies did not. Given these differences it is remarkable that so many of the results are as similar as they are.

Because Factor 1 accounted for the majority of the variance in the sample, it is worth examining more closely. Factor 1 of the current study includes coprophenomena (coprolalia, copropraxia, mental coprolalia), echophenomena (echolalia, echopraxia), paliphenomena (palilalia, palipraxia), random words, as well as forced touching (of other people and/or external objects), hitting, kicking and spitting. This could be argued to be a 'socially inappropriate factor', similar to the Alsobrook \& Pauls ${ }^{23}$ Factor 1, which included coprolalia, aggressive and self-injurious behaviour, all of which are socially inappropriate. It is important to note that copropraxia, mental coproprolalia and palipraxia were not assessed in the Alsobrook \& Pauls ${ }^{23}$ study. There may be some similarity to the Robertson \& Cavanna ${ }^{25}$ Factor 2, which was predominantly ADHD and aggressive behaviours. Attentiondeficit hyperactivity disorder was not included in the factor analysis of the present study, but, interestingly, factor scores on Factor 1 were significantly higher in the subset of the sample with ADHD as well as individuals with a family history of ADHD. The major difference between the current study and the two previous principal components factor analysis studies ${ }^{23,25}$ is that in the present study, echophenomena (echolalia, echopraxia), paliphenomena and random words also formed part of Factor 1, but were not part of this factor in either the Alsobrook \& Pauls ${ }^{23}$ or Robertson \& Cavanna ${ }^{25}$ studies. Of note and particular historical importance is that Georges Gilles de la Tourette ${ }^{31}$ described a syndrome emphasising a triad of multiple motor tics, coprolalia and echolalia, which is very similar to our Factor 1.

\section{Self-injury and Tourette syndrome}

Georges Gilles de la Tourette also considered self-injurious behaviour as an important component, as has recent research. ${ }^{32,33}$ In the current cluster analyses and those of Mathews et $a l^{24}$ and Alsobrook \& Pauls, ${ }^{23}$ self-injurious behaviour resides in the same broad cluster as other complex symptoms such as copro- pali- and echophenomena. It is also an important symptom encountered in individuals with mild Tourette syndrome such as those previously undiagnosed and not under medical care for their symptoms, ${ }^{26}$ and has been shown to be intimately and significantly related to both obsessionality ${ }^{32}$ and impulsivity. ${ }^{33}$ It is therefore possible, indeed probable, that there are different types of self-injurious behaviour, some more aggressive and others more compulsive, which could account for the differential loading in the different studies as well as the fact that self-injurious behaviour is in both Factor 1 and Factor 4 in the current results. Furthermore, it is also possible that the manner in which self-injurious behaviour is perceived or stressed in the interviews may have been different or there may be cultural differences across the samples in their interpretation or expression of this behaviour. Nevertheless, it is consistent with a certain 'face validity' of self-injurious behaviour in Tourette syndrome that it be variably grouped as either aggressive or compulsive, as it appears to be both.

That the socially inappropriate behaviours and compulsivelike tics were observed to be associated with different co-occurring psychopathology raises the hope that there may be consistent, dissociable and potentially genetically informative phenotypes within Tourette syndrome which may enhance genotypic examinations. Moreover, as both genetic and environmental factors have been demonstrated in aggressive ${ }^{34}$ and compulsive-like ${ }^{35}$ phenomena, these data also raise the possibility of enhancing the yield of exploration into gene $v$. environment interactions for Tourette syndrome as these environmental factors, important for aggressive and compulsive phenomena, become better characterised.

\section{Summary of conclusions and limitations}

The results of the current research and those of the previously published studies, although not identical, suggest that the phenotype of Tourette syndrome is complex. Thus, one distinct component encountered in Tourette syndrome includes tics alone which may be either simple or complex. A second component appears to consist of behaviours that may best be classified as 'socially inappropriate' (which in some cases could be argued to be aggressive), including coprolalia, mental coprolalia, copropraxia, spitting, hitting and kicking and, in the current study, the more complex vocal/phonic tics such as echolalia and palilalia. Put together, these two components are what were originally described by Georges Gilles de la Tourette in $1885 .{ }^{31}$ A statistically separate component appears to consist of behaviours that are best categorised as compulsive-like, including forced touching and repetitive looking at objects (e.g. checking their whereabouts) and other ritualised behaviours.

It is also clear from all studies that there is additional phenotypic variance that is not accounted for by the factors described in the analyses. Nevertheless, the similarity between all studies suggests that there are certain clusters of tics that may occur together more often than expected by chance. Furthermore, some of these factors may be uniquely heritable. ${ }^{23}$ 
Limitations of our study include the fact that it was not identical to any previous study with regard to data or methods used. For example, the NHIS did not collect identical information or measures used in the Alsobrook \& Pauls ${ }^{23}$ study (with similar methods), so a direct comparison between the studies was not possible. The data collected were, however, similar to Robertson \& Cavanna, ${ }^{25}$ but the principal components factor analysis methods were different and their study was performed on a larger extended pedigree, whereas our study was on people in the clinic setting.

Mary M. Robertson, MBChB, MD, DSC (Med), FRCPCH, FRCP (UK), FRCPsych, Department of Mental Health Sciences, University College London, London, UK, Robert R. Althoff, MD, PhD, Department of Psychiatry, University of Vermont College of Medicine, Burlington, Vermont, Canada; Adam Hafez, Medical Student, King's College London, London, UK; David L. Pauls, PhD, Psychiatric and Neurodevelopmental Genetics Unit, Center for Human Genetic Research, Massachusetts General Hospital, Harvard Medical School, Boston, Massachusetts, USA

Correspondence: David Pauls, Psychiatric and Neurodevelopmental Genetics Unit, Center for Human Genetic Research, Massachusetts General Hospital, Harvard Medical School, 185 Cambridge Street, Boston, MA 02114 USA. Email: dpauls@pngu.mgh.harvard.edu

First received 1 May 2007, final revision 3 Feb 2008, accepted 15 Feb 2008

\section{Acknowledgements}

We thank Rachel Carter and Elizabeth Cowan for entering data. We also acknowledge the helpful critiques of two anonymous reviewers. Their suggestions significantly improved the manuscript. The work was funded by NIH Grants NS16648 and NS40024 (D.L.P.) and The National Hospital for Neurology and Neurosurgery Development Foundation (M.M.R.)

\section{References}

1 World Health Organization. International Classification of Diseases, 10th Edition (ICD-10). WHO, 1992.

2 American Psychiatric Association. Diagnostic and Statistical Manual of Mental Disorders (4th edn - Text Revision) (DSM-IV-TR). APA, 2000.

3 Pauls DL. An update on the genetics of Gilles de la Tourette syndrome. J Psychosom Res 2003; 55: 7-12.

4 Price RA, Kidd KK, Cohen DJ, Pauls DL, Leckman JF. A twin study of Tourette syndrome. Arch Gen Psychiatry 1985; 42: 815-20.

5 Hyde TM, Aaronson BA, Randolph C, Rickler KC, Weinberger DR. Relationship of birth weight to the phenotypic expression of Gilles de la Tourette's syndrome in monozygotic twins. Neurology 1992; 42: 652-8.

6 Curtis D, Robertson MM, Gurling HM. Autosomal dominant gene transmission in a large kindred with Gilles de la Tourette syndrome. Br J Psychiatry 1992; 160: $845-9$

7 Tourette Syndrome Association International Consortium for Genetics. A complete genome screen in sib pairs affected by Gilles de la Tourette syndrome. Am J Hum Genet 1999; 65: 1428-36.

8 Barr CL, Wigg KG, Pakstis AJ, Kurlan R, Pauls D, Kidd KK, Tsui LC, Sandor P. Genome scan for linkage to Gilles de la Tourette syndrome. Am J Med Genet 1999; 88: 437-45.

9 Mérette C, Brassard A, Potvin A, Bouvier H, Rousseau F, Emond C, Bissonnette L, Roy MA, Maziade M, Ott J, Caron C. Significant linkage for Tourette syndrome in a large French Canadian family. Am J Hum Genet 2000; 67: 1008-13.

10 Simonic I, Nyholt DR, Gericke GS, Gordon D, Matsumoto N, Ledbetter DH, Ot J, Weber JL. Further evidence for linkage of Gilles de la Tourette syndrome (GTS) susceptibility loci on chromosomes 2p11, 8q22 and 11q23-24 in South African Afrikaners. Am J Med Genet 2001; 105: 163-7.

11 Zhang $\mathrm{H}$, Leckman JF, Pauls DL, Tsai CP, Kidd KK, Campos MR, Tourette Syndrome Association International Consortium for Genetics. Genomewide scan of hoarding in sib pairs in which both sibs have Gilles de la Tourette syndrome. Am J Hum Genet 2002; 70: 896-904.

12 Curtis D, Brett P, Dearlove AM, McQuillin A, Kalsi G, Robertson MM, Gurling HM. Genome scan of Tourette syndrome in a single large pedigree shows some support for linkage to regions of chromosome 5, 10 and 13. Psychiatr Genet 2004; 14: 83-7.

13 Paschou $P$, Feng $Y$, Pakstis AJ, Speed WC, DeMille MM, Kidd JR, Jaghori $B$, Kurlan R, Pauls DL, Sandor P, Barr CL, Kidd KK. Indications of linkage and association of Gilles de la Tourette syndrome in two independent family samples: $17 q 25$ is a putative susceptibility region. Am J Hum Genet 2004; 75 $545-60$.
14 Abelson JF, Kwan KY, O'Roak BJ, Baek DY, Stillman AA, Morgan TM Mathews CA, Pauls DL, Rasin MR, Gunel M, Davis NR, Ercan-Sencicek AG, Guez DH, Spertus JA, Leckman JF, Dure LS 4th, Kurlan R, Singer HS, Gilbert $\mathrm{DL}$, Farhi A, Louvi A, Lifton RP, Sestan N, State MW. Sequence variants in SLITRK1 are associated with Tourette's Syndrome. Science 2005; 310: $317-20$

15 Tourette Syndrome Association International Consortium for Genetics. Genome scan for Tourette disorder in affected-sibling-pair and multigenerational families. Am J Hum Genet 2007; 80: 265-72.

16 Grigorenko EL, Wood FB, Meyer MS, Hart LA, Speed WC, Shuster A, Pauls DL. Susceptibility loci for distinct components of developmental dyslexia on chromosomes 6 and 15. Am J Hum Genet 1997; 60: 27-39.

17 Todd RD, Rasmussen ER, Neuman RJ, Reich W, Hudziak JJ, Bucholz KK, Madden PA, Heath A. Familiality and heritability of subtypes of attention deficit hyperactivity disorder in a population sample of adolescent female twins. Am J Psychiatry 2001; 158: 1891-8.

18 Neuman RJ, Heath A, Reich W, Bucholz KK, Madden PAF , Sun L, Todd RD Hudziak JJ. Latent class analysis of ADHD and comorbid symptoms in a population sample of adolescent female twins. I Child Psychol Psychiatry 2001; 42: 933-42.

19 Alsobrook II JP, Leckman JF, Goodman WK, Rasmussen SA, Pauls DL. Segregation analysis of obsessive-compulsive disorder using symptom-based factor scores. Am J Med Genet 1999; 88: 669-75.

20 Bradford $Y$, Haines J, Hutcheson $H$, Gardiner $M$, Braun T, Sheffield V, Cassavant T, Huang W, Wang K, Vieland V, Folstein S, Santangelo S, Piven J. Incorporating language phenotypes strengthens evidence of linkage to autism. Am J Med Genet 2002; 10: 539-47.

21 Jagger J, Prusoff BA, Cohen DJ, Kidd KK, Carbonari CM, John K. The epidemiology of Tourette's syndrome: a pilot study. Schizophr Bull 1982: 8 267-78.

22 Robertson MM, Trimble MR, Lees AJ. The psychopathology of the Gilles de la Tourette syndrome. A phenomenological analysis. Br J Psychiatry 1988; 152: 383-90.

23 Alsobrook, JP II, Pauls DL. A factor analysis of tic symptoms in Gilles de la Tourette syndrome. Am J Psychiatry 2002; 159: 291-6.

24 Mathews $C A$, Jang $\mathrm{KL}$, Herrera $\mathrm{LD}$, Lowe $\mathrm{TL}$, Budman $\mathrm{CL}$, Erenberg $\mathrm{G}$, Naarden A, Bruun RD, Schork NJ, Freimer NB, Reus VI. Tic symptom profiles in subjects with Tourette syndrome from two genetically isolated populations. Biol Psychiatry 2007; 61: 292-300.

25 Robertson MM, Cavanna AE. The Gilles de la Tourette syndrome: a principal component factor analytic study of a large pedigree. Psychiatr Gene 2007; 17: $143-52$.

26 Robertson MM, Gourdie A. Familial Tourette's syndrome in a large British pedigree. Associated psychopathology, severity, and potential for linkage analysis. Br J Psychiatry 1990; 156: 515-21.

27 Verkerk AJHM, Cath DC, van der Linde HC, Both J, Heutink P, Breedveld G, Aulchenko YS, Oostra BA. Genetic and clinical analysis of a large Dutch Gilles de la Tourette family. Mol Psychiatry 2006; 10: 954-64.

28 American Psychiatric Association. Diagnotistic and Statistical Manual of Mental Disorders (3rd edn) (DSM-III). APA, 1980.

29 Robertson MM, Eapen V. The National Hospital Interview Schedule for the assessment of Gilles de la Tourette syndrome and related behaviors Int J Methods Psychiatr Res 1996; 6: 203-26.

30 Leckman JF, Riddle MA, Hardin MT, Ort SI, Swartz KL, Stevenson J, Cohen DJ. The Yale Global Tic Severity Scale: initial testing of a clinician-rated scale of tic severity. J Am Acad Child Adolesc Psychiatry 1989; 28: 566-73.

31 Gilles de la Tourette, G. Etude sur une affection nerveuse caracterise par de l'incoordination motrice accompagnee d'echolalie et de coproalalie [Study of a nervous disorder characterised by motor incoordination accompanied by echolalia and coprolalia]. Arch Neurol (Paris) 1885; 19-42: 158-200.

32 Robertson MM, Trimble MR Lees AJ. Self injurious behaviour and The Gilles de la Tourette Syndrome. A clinical study and review of the literature. Psychol Med 1989; 19: 611-25.

33 Mathews CA, Waller J, Glidden D, Lowe TL, Herrera LD, Budman CL, Erenberg G, Naarden A, Bruun RD, Freimer NB, Reus VI. Self injurious behaviour in Tourette syndrome: correlates with impulsivity and impulse control. I Neurol Neurosurg Psychiatry 2004; 75: 1149-55.

34 van Beijsterveldt CE, Bartels M, Hudziak JJ, Boomsma DI. Causes of stability of aggression from early childhood to adolescence: a longitudinal genetic analysis in Dutch twins. Behav Genet 2003; 33: 591-605.

35 Hudziak JJ, Van Beijsterveldt CE, Althoff RR, Stanger C, Rettew DC, Nelson $\mathrm{EC}$, Todd RD, Bartels M, Boomsma DI. Genetic and environmental contributions to the Child Behavior Checklist Obsessive-Compulsive Scale: a cross-cultural twin study. Arch Gen Psychiatry 2004; 61: 608-16. 